\title{
Divergence type of some subgroups of finitely generated Fuchsian groups
}

\author{
MARY REES † \\ From the Institut des Hautes Etudes Scientifiques, Bures-sur-Yvette, France
}

(Received 13 November 1980)

\begin{abstract}
Let $\Gamma$ be a finitely generated discrete subgroup of the isometries of the hyperbolic plane $H^{2}$ with at least one parabolic element. We prove that, if $\Gamma_{1}$ is a subgroup of $\Gamma$ with $\Gamma / \Gamma_{1}$ abelian, the 'critical exponent' of $\Gamma_{1}$ is the same as that of $\Gamma$. We give necessary and sufficient conditions - in terms of the rank of $\Gamma / \Gamma_{1}$, the critical exponent of $\Gamma$, and the image of parabolic elements of $\Gamma$ in $\Gamma / \Gamma_{1}-$ for the Poincaré series of $\Gamma_{1}$ to diverge at the critical exponent.
\end{abstract}

\section{Introduction}

The aim of this paper is to determine when a subgroup $\Gamma_{1}$ of a finitely generated discrete group of isometries $\Gamma$ of 2-dimensional hyperbolic space $H^{2}$, with $\Gamma_{1} \triangleleft \Gamma$, $\Gamma / \Gamma_{1}$ abelian, is of divergence type [8], i.e. whether or not the series

$$
\sum_{y \in \Gamma_{1}} \exp \{-\alpha(x, \gamma x)\}
$$

diverges at $\alpha=\delta\left(\Gamma_{1}\right)$, for $\delta\left(\Gamma_{1}\right)$ the supremum of the $\alpha$ for which the series diverges, where $x \in H^{2}$ is any fixed point and $(x, \gamma x)$ denotes hyperbolic distance between $x$ and $\gamma x$. Equivalently (for $\delta\left(\Gamma_{1}\right)>\frac{1}{2}$ ), we wish to determine whether the geodesic flow $\left\{\phi_{t}\right\}$ on the unit tangent bundle UT $\left(H^{2} / \Gamma_{1}\right)$ of $H^{2} / \Gamma_{1}$ is ergodic with respect to certain natural measures [8]. We restrict our discussion to the case of $H^{2} / \Gamma$ having cusps. The case of $H^{2} / \Gamma$ without cusps was dealt with in [6].

Recently, Lyons \& McKean [2] proved that, if $\Gamma$ is the fundamental group of the thrice-punctured sphere, and $\Gamma_{1}$ is the commutator subgroup of $\Gamma$, then hyperbolic Brownian motion on $H^{2} / \Gamma_{1}$ is not recurrent, and that $\Gamma_{1}$ is not of divergence type (equivalent conditions by [8]) (see also [3]). This is the sort of example we have in mind. However, we do not restrict the critical exponent of $\Gamma_{1}$ to be 1 (as in the cited example). The main results are summarized in theorem 1.

THEOREM 1. Let $\Gamma$ be a discrete finitely generated group of isometries of $H^{2}$. Let $\Gamma_{1} \triangleleft \Gamma, \Gamma / \Gamma_{1} \simeq \mathbb{Z}^{v}$, and let $\boldsymbol{\theta}: \Gamma \underset{\text { onto }}{\longrightarrow} \mathbb{Z}^{v_{1}}, \boldsymbol{\phi}: \Gamma \underset{\text { onto }}{\longrightarrow} \mathbb{Z}^{v_{2}}\left(v=v_{1}+v_{2}\right)$ be two homomorphisms with $\Gamma_{1}=\operatorname{Ker}(\theta \oplus \phi)$, where, if $x_{1} \cdots x_{r}$ are parabolic elements of $\Gamma$ corresponding to the $r$ cusps of $H^{2} / \Gamma\left(x_{1} \cdots x_{r}\right.$ are unique up to taking inverses, and 
conjugation $)$, then $\boldsymbol{\phi}\left(x_{i}\right)=0$ for all $i$, and $\left\langle\boldsymbol{\theta}\left(x_{1}\right), \ldots, \boldsymbol{\theta}\left(x_{r}\right)\right\rangle$ is syndetic in $\mathbb{Z}^{v_{1}}$. (Clearly any homomorphism onto $\mathbb{Z}^{v}$ can be put in the form $\theta \oplus \phi$.) Then $\delta(\Gamma)=\delta\left(\Gamma_{1}\right)=\delta$, say.

If $\delta<1, \Gamma_{1}$ is of divergence type if and only if $v_{1}=0$ and $v_{2} \leq 2$. If $\delta=1, \Gamma_{1}$ is of divergence type if and only if either $v_{1} \leq 1$ and $v_{2}=0$, or $v_{1}=0$ and $v_{2} \leq 2$.

The proof uses symbolic dynamics and the same general method, with modification, as in [6]. Because there is no developed theory of symbolic dynamics for cusped manifolds in higher dimensions, the methods only work in dimension 2. However, as in [6], the results can be extended to 'finitely determined sub-abelian' subgroups of $\Gamma$. We do not give the details here (nor the definitions) - the analogue with $\S 5$ of [6] is fairly exact. But, for example, if $H^{2} / \Gamma$ is a 1 -cusped surface of genus $g \geq 1$, with $a_{1} \cdots a_{\mathrm{g}}, b_{1} \cdots b_{\mathrm{g}}$ the free generators of $\Gamma$ and $\prod_{i=1}^{\mathrm{g}}\left[a_{i}, b_{i}\right]$ representing the cusp, where $\left[a_{i}, b_{i}\right]=a_{i} b_{i} a_{i}^{-1} b_{i}^{-1}$, and if

$$
\begin{aligned}
\Gamma_{1}= & \left\{\text { words in }\left\{a_{i}, b_{i}, a_{i}^{-1}, b_{i}^{-1} ; i=1, \ldots, g\right\}: \text { sum of } a_{1}\right. \text {-powers } \\
& \left.=\text { sum of } b_{1} \text {-powers }=0\right\},
\end{aligned}
$$

so that

$$
\mathscr{F}=\bigcup_{i=2}^{g}\left\{a_{i}, b_{i}, a_{i}^{-1}, b_{i}^{-1}\right\} \cup \bigcup_{m, n \in \mathbb{Z} \backslash\{0\}}\left(a_{1}^{m} b_{1}^{n} a_{1}^{-m} b_{1}^{-n}, b_{1}^{n} a_{1}^{m} b_{1}^{-n} a_{1}^{-m}\right\}
$$

is a set of free generators and inverses of generators of $\Gamma_{1}$, and if

$$
\Gamma_{2}=\left\{\text { words in the elements of } \mathscr{F} \text { : sum of }\left[a_{1}, b_{1}\right] \text {-powers }=0\right\} \text {, }
$$

then the generalized theorem 1 gives $\Gamma_{2}$ is of divergence type with $\delta(\Gamma)=\delta\left(\Gamma_{2}\right)=1$.

\section{Formulation of the symbolic dynamics}

In some ways, the presence of a cusp on $H^{2} / \Gamma$ actually makes things easier. Throughout this paper, we assume without loss of generality that no $\gamma \in \Gamma$ fixes any points of $H^{2}$, so that $H^{2} / \Gamma$ is a manifold with $\Gamma$ as its fundamental group. Since we assume $H^{2} / \Gamma$ has at least one cusp, $H^{2} / \Gamma$ is topologically either a $k$-holed sphere $(k \geq 3)$ or a $k$-holed surface of genus $\geq 1(k \geq 1)$. So $\Gamma$ must be a free group, and $H^{2} / \Gamma$ has a geodesic triangulation - i.e. the edges of the triangulation are geodesics - with vertices only at the holes. (Note that, besides one or more cusps, some of the 'holes' may be ends of infinite volume.) The point of this is that $H^{2} / \Gamma$ has a fundamental region $F$ on $H^{2}$, bounded by geodesic arcs and arcs on the boundary of $H^{2}$ (possibly) with all corners on the boundary, and the images of $F$ under $\Gamma$ which are adjacent to $F$ are $a_{1} F, a_{2} F, \ldots, a_{s} F, a_{1}^{-1} F, \ldots, a_{s}^{-1} F$, for some free generating set $\left\{a_{1} \cdots a_{s}\right\}$ of $\Gamma$. For instance, in the example in figure $1, F$ is the fundamental region of a 3 -holed sphere with 2 cusps and 1 infinite volume end.

Patterson [5] proves that any finitely generated group $\Gamma$ is of divergence type. By [1] the presence of a cusp means the critical exponent $\delta(\Gamma)$ satisfies $\frac{1}{2}<\delta(\Gamma) \leq 1$. Equivalently to the divergence type condition, the geodesic flow (UT $\left.\left(H^{2} / \Gamma\right),\left\{\phi_{t}\right\}, \mu_{\nu}\right)$ is ergodic, where $\mu_{\nu}$ is the geodesic-flow invariant measure on $\mathrm{UT}\left(H^{2} / \Gamma\right)$ naturally corresponding to the (unique) $\Gamma$-invariant conformal density $\nu$ of dimension $\delta(\Gamma)$ on the limit set $L_{\Gamma}$ of $\Gamma[8]$. 


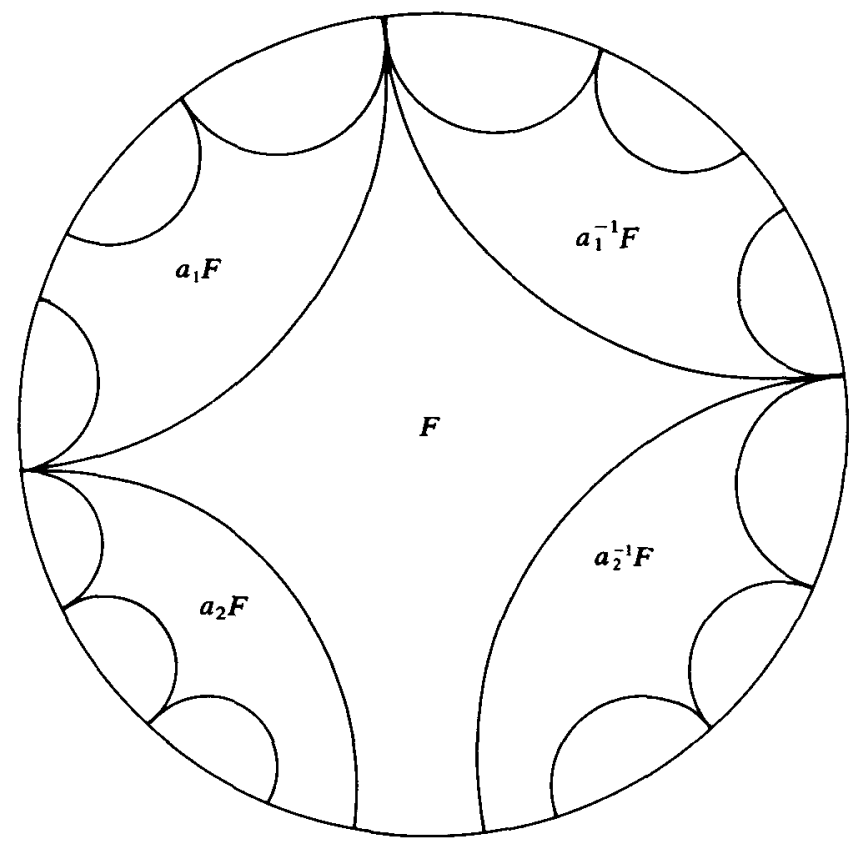

Figure 1

We now describe a 'subshift of finite type' $(Y, \sigma)$ on an infinite set of symbols $\mathscr{K}=\mathscr{K}^{-1} \subseteq \Gamma$ such that (UT $\left.\left(H^{2} / \Gamma\right),\left\{\phi_{t}\right\}, \mu_{\nu}\right)$ is measure-theoretically isomorphic to the suspension under a positive (unbounded) function of a Gibbs $\sigma$-invariant probability measure (also denoted $\mu_{\nu}$ ) on $Y . \sigma$ is defined on $Y \subseteq \mathscr{K}^{\mathbf{Z}}$ by $\sigma\left(\left\{x_{i}\right\}\right)=$ $\left\{x_{i+1}\right\} . Y$ is also invariant under $\tau$, where $\tau\left(\left\{x_{i}\right\}\right)=\left\{x_{-i}^{-1}\right\}$, and $\tau_{*} \mu_{\nu}=\mu_{\nu}$.

The symbols of $\mathscr{K}$ are obtained as follows. Each cusp vertex $Z_{i}$ of $F$ is fixed by a unique (up to taking its inverse) parabolic element of $\Gamma$, which is a word of minimal length $n_{i}$ in $\left\{a_{1} \cdots a_{s}, a_{1}^{-1} \cdots a_{s}^{-1}\right\}$. Choose $N>1$ such that $n_{i} \mid N, i=1 \cdots r$ (if $r$ is the number of cusps on $F$ ). Let $c_{1} \cdots c_{r}, c_{1}^{-1} \cdots c_{r}^{-1}$ denote the parabolic elements of length $N$ fixing the cusps of $F$, and $d_{1} \cdots d_{t}, d_{1}^{-1} \cdots d_{t}^{-1}$ the other words of length $N$. Suppose a geodesic in $H^{2}$ passes successively through $g_{N}^{-1} g_{N-1}^{-1} \cdots g_{1}^{-1} F, g_{N}^{-1} g_{N-1}^{-1} \cdots g_{2}^{-1} F, \ldots, g_{N}^{-1} F, F, h_{1} F, h_{1} h_{2} F, \ldots, h_{1} h_{2} \cdots h_{N} F$, where $g_{1} \cdots g_{N}, h_{1} \cdots h_{N}$ are words of length $N$ in $\left\{a_{1} \cdots a_{s}, a_{1}^{-1} \cdots a_{s}^{-1}\right\}$ (so $g_{i} \neq g_{i+1}^{-1}$, $\left.h_{i} \neq h_{i+1}^{-1}\right)$. Then $g_{N}^{-1} \neq h_{1}$. Moreover, the geodesic has endpoints a Euclidean distance apart bounded away from zero (in the disk model for $H^{2}$ ) unless $g_{1} \cdots g_{N}=$ $h_{1} \cdots h_{N}=c$, where $c=c_{i}$ or $c_{i}^{-1}$, some $i=1, \ldots, r$. Then

$$
\begin{gathered}
\mathscr{K}=\bigcup_{i=1}^{r} \bigcup_{n \in Z \backslash\{0\}}\left\{c_{i}^{n}\right\} \cup\left\{d_{1} \cdots d_{t}, d_{1}^{-1} \cdots d_{t}^{-1}\right\} \\
Y=\left\{\left\{x_{i}\right\} \in \mathscr{K}^{\mathbf{Z}}: x_{i} x_{i+1} \text { is admissible }\right\}
\end{gathered}
$$

where admissibility is defined by the following: ef is admissible if and only if

(i) the last element of $\left\{a_{1} \cdots a_{s}, a_{1}^{-1} \cdots a_{s}^{-1}\right\}$ in the word $e$ is not the inverse of the first element of the word $f$; 
(ii) if $e=c_{k}^{n}$, some $k, n$, then $f \neq c_{k}^{m}$, any $m$.

The realization of (UT $\left.\left(H^{2} / \Gamma\right),\left\{\phi_{t}\right\}, \mu_{\nu}\right)$ as a suspension of $\left(Y, \sigma, \mu_{\nu}\right)$ is as follows. $\mu_{\nu}$-almost all geodesics in $H^{2} / \Gamma$ have a unique lift in $H^{2}$ passing through a bi-infinite sequence of fundamental regions

$$
\cdots f_{-1}^{-1} f_{-2}^{-1} F, f_{-1}^{-1} F, F, f_{0} F, f_{0} f_{1} F, f_{0} f_{1} f_{2} F, \cdots
$$

where $\quad f_{i} \in\left\{a_{1} \cdots a_{s}, a_{1}^{-1} \cdots a_{s}^{-1}\right\}$ and $f_{i+1} \neq f_{i}^{-1}$ for any $i$.

These symbols $\cdots f_{-1}, f_{0}, f_{1} \cdots$ can be uniquely grouped into words of length $N$ in $\left\{a_{1} \cdots a_{s}, a_{1}^{-1} \cdots a_{s}^{-1}\right\}$ such that $f_{0}$ is the first element of its word. The symbols of one of the new sequences $\left\{g_{i}\right\}$ come from $\left\{c_{1} \cdots c_{r}, c_{1}^{-1} \cdots c_{r}^{-1}\right\} \cup\left\{d_{1} \cdots d_{t}, d_{1}^{-1} \cdots d_{t}^{-1}\right\}$. Now the symbols $\left\{g_{i}\right\}$ can be uniquely regrouped to give a bi-infinite sequence of elements of $\mathscr{K}$. The set of sequences thus obtained is residual in $Y$ and, of course, has full $\mu_{\nu}$-measure in $Y$. See also Series [7].

The measure $\mu_{\nu}$ on $Y$ is Gibbs in the sense of [6]. The proof is the same as in [6], using the fact stated here that if a geodesic passes successively through $e^{-1} F, F, f F$, where, in the regrouping, $e$ is the last symbol of a word of $\mathscr{K}$ and $f$ the first symbol of another word of $\mathscr{K}$, then the endpoints are a Euclidean distance apart bounded away from zero. Also lemma 2 is used to show finiteness of the measure $\mu_{\nu}$ on $Y$ (and also something more, to be used later). Since $\mu_{\nu}$ is Gibbs, it has a very good approximation by Markov measures and the same general method as [6] might work.

LEMMA 2. There exists a constant $b_{i}>0$ such that

$$
\mu_{\nu}\left(\left[c_{i}^{n}\right]\right)=\frac{b_{i}}{|n|^{2 \delta}}\left(1+O\left(\frac{1}{|n|}\right)\right)
$$

where $\nu$ is a $\Gamma$-invariant conformal density of dimension $\delta$-on $L_{\Gamma}\left(\right.$ i.e. $d \gamma_{*} \nu(\xi) / d \nu=$ $\left|\gamma^{\prime}(\xi)\right|^{\delta}, \xi \in L_{\Gamma}$, where $\left.\gamma_{*} \nu(f)=\nu\left(f \circ \gamma^{-1}\right)\right)$ and $\left[c_{i}^{n}\right]=\left\{\left\{x_{j}\right\} \in Y: x_{0}=c_{i}^{n}\right\}$.

Proof. $Y$ can be identified measure-theoretically with the set of $(\xi, \eta) \in L_{\Gamma} \times L_{\Gamma}$ for which the geodesic from $\xi$ to $\eta$ passes through the interior of $F$, and then $\mu_{\nu}$ identifies with the measure $d \nu(\xi) d \nu(\eta) /|\xi-\eta|^{2 \delta}$ on this subset of $L_{\Gamma} \times L_{\Gamma}$, with $\nu$ suitably normalized [8]. [ $c_{i}^{n}$ ] then identifies with $U \times c_{i}^{n-1} V$ as shown, for $n \geq 1$, in figure 2 . (The case $n \leq-1$ is similar. Recall that $c_{i} F$ is the $N$ th region round the cusp from $F$, so not next to $F$, since $N>1$.)

$U$ is bounded away from $\bigcup_{n \geq 0} c_{i}^{n} V$. Let $\eta_{0}$ be the fixed point of $c_{i}$. The maximum distance between $\eta_{0}$ and $c_{i}^{n} V$ (Euclidean distance for the disk) is $O\left(1 / n^{2}\right)$ (since this is the size of the derivative of $c_{i}^{n}$ over most of $S^{1}$ ). So

$$
\left|\mu_{\nu}\left(\left[c_{i}^{n}\right]\right)-\nu\left(c_{i}^{n-1} V\right) \int_{U} \frac{d \nu(\xi)}{\left|\xi-\eta_{0}\right|^{2 \delta}}\right| \leq \frac{A \nu\left(c_{i}^{n-1} V\right)}{n^{2}}
$$

(some $\boldsymbol{A}$ ). Since $\nu$ is a conformal density,

$$
\nu\left(c_{i}^{n-1} V\right)=\int \chi_{V}\left(c_{i}^{-(n-1)} \xi\right) d \nu(\xi)=\int \chi_{V}(\xi)\left|\left(c_{i}^{n-1}\right)^{\prime}(\xi)\right|^{\delta} d \nu(\xi) .
$$

Let $x$ be a conformal map of the disk to the upper half plane, mapping $c_{1}$ to a parabolic transformation fixing 0 , and $V$ to the interval $[1,2] \subseteq \mathbb{R}$, and let $\nu_{1}$ be the 


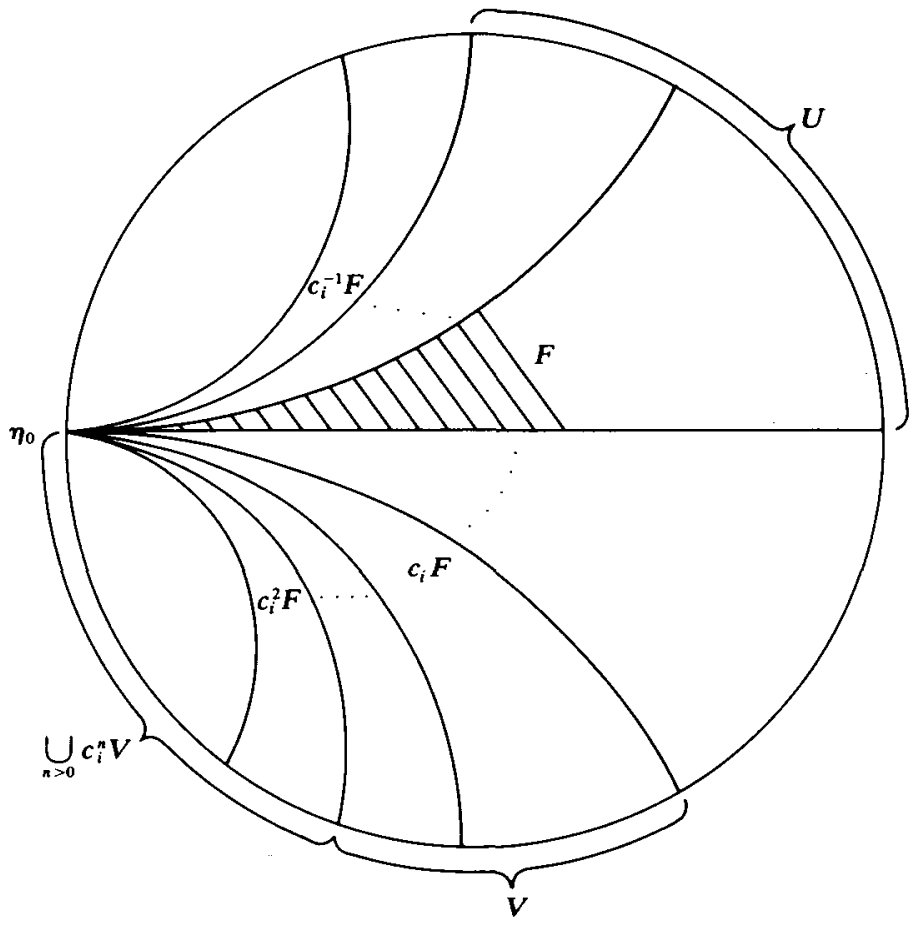

Figure 2

measure on $\mathbb{R} \cup\{\infty\}$ given by $\nu_{1}(f)=\nu(f \circ x)$. Then

$$
\nu\left(c_{i}^{n-1} V\right)=\int_{1}^{2} \frac{g(x)}{(1+a(n-1) x)^{2 \delta}} d \nu_{1}(x)
$$

for some $a>0$ and some smooth positive function $g$. Then

$$
\nu\left(c_{i}^{n-1} V\right)=\frac{1}{n^{2 \delta}}\left\{\int_{1}^{2} \frac{g(x)}{(a x)^{2 \delta}} d \nu_{1}(x)+O(1 / n)\right\}
$$

as required.

Given the symbolic dynamics, we can now reformulate theorem 1 , using the notation of theorem 1. The proof of (1) of theorem 3 is as in $\$ \S 1,2$ of [6].

THEOREM 3. (1) Let $S_{k}=\sum\left\{\mu_{\nu}\left(\left[e_{0} \cdots e_{k-1}\right]: e_{i} \in \mathscr{K},\left[e_{0} \cdots e_{k-1}\right] \subseteq Y\right.\right.$ and $\neq \varnothing$, and $e_{0} e_{1} \cdots e_{k-1} \in \Gamma_{1}$ i.e. $\left.(\boldsymbol{\theta}+\boldsymbol{\phi})\left(e_{0} e_{1} \cdots e_{k-1}\right)=0\right\}$ where

$$
\Gamma_{1} \leq \Gamma \text { and }\left[e_{0} \cdots e_{k-1}\right]=\left\{\left\{x_{i}\right\} \in Y: x_{i}=e_{i}, 0 \leq i \leq k-1\right\} \text {. }
$$

Then (UT $\left.\left(H^{2} / \Gamma_{1}\right),\left\{\phi_{t}\right\}, \mu_{\nu}\right)$ is ergodic if and only if $\sum_{k=1}^{\infty} S_{k}=\infty$, where $\mu_{\nu}$ is the unique measure on UT $\left(H^{2} / \Gamma_{1}\right)$ such that local inverses of the natural projection onto (UT $\left.\left(H^{2} / \Gamma\right), \mu_{\nu}\right)$ are measure-preserving. Equivalently, $\Gamma_{1}$ is of divergence type if and only if $\sum_{k=1}^{\infty} S_{k}=\infty$. 
(2) There exist constants $A, B>0$ such that

$$
\frac{A}{k^{v_{i}(2 \delta-1)+\frac{1}{2} v_{2}}} \leq S_{k}+S_{k+1} \leq \frac{B}{k^{v_{i} / 2(\delta-1)+\frac{1}{2} v_{2}}}
$$

where $\delta=\delta(\Gamma)$.

Note. Since theorem 3 implies $S_{k}+S_{k+1} \geq c \sum_{\gamma \in A_{k} \leq \Gamma} \exp \{-\delta(x, \gamma x)\}$ for some constant $c>0$, and $\Gamma_{1}=\bigcup_{k=1}^{\infty} A_{k}$ a disjoint union with $A_{k} \subseteq\{\gamma:(x, \gamma x) \geq d k\}$, some constant $d$ (essentially the same proof as 1.10 of [6]), $\delta\left(\Gamma_{1}\right)=\delta=\delta(\Gamma)$.

The proof of theorem 3 . In modifying the proof of 4.7 in [6] to prove theorem 3 , we have first to recall some notation, and define some new notation.

Notation. (1) Let $\varepsilon_{m}$ denote the set of non-empty $m$-cylinders $\left[e_{0} \cdots e_{m-1}\right]$ of $Y$, where

$$
\left[e_{0} \cdots e_{m-1}\right]=\left\{\left\{x_{i}\right\} \in Y: x_{i}=e_{i}, 0 \leq i \leq m-1\right\}
$$

for $e_{i} \in \mathscr{K}$. $\varepsilon_{m}$ can be regarded as a subset of $\Gamma$ (by multiplying the symbols) so the homomorphisms $\boldsymbol{\theta}, \boldsymbol{\phi}$ are defined on $\varepsilon_{m}$,

$$
\boldsymbol{\theta} \oplus \boldsymbol{\phi}: \varepsilon_{m_{-}} \rightarrow \bigoplus_{i=1}^{v_{1}}\left\langle\theta_{i}\right\rangle \oplus \bigoplus_{i=1}^{v_{2}}\left\langle\phi_{i}\right\rangle
$$

(2) $W_{m}$ is a row vector, $A_{m}(\theta, \phi)$ is a matrix, $V_{m}(\theta, \phi)$ is a column vector, rows and columns are indexed by $\varepsilon_{m}$.

$$
\begin{aligned}
W_{m}(\mathbf{c}) & =1 \quad \text { for all } \mathbf{c} \in \varepsilon_{m}, \\
V_{m}(\boldsymbol{\theta}, \boldsymbol{\phi})(\mathbf{c}) & =\mu_{\nu}(\mathbf{c}) \exp \{i(\boldsymbol{\theta}+\boldsymbol{\phi})(\mathbf{c})\}, \\
\boldsymbol{A}_{m}(\boldsymbol{\theta}, \boldsymbol{\phi})(\mathbf{c}, \mathbf{d}) & =\exp \left\{i(\boldsymbol{\theta}+\boldsymbol{\phi})\left(c_{m-1}\right)\right\} \cdot\left\{\frac{\mu_{\nu}\left(\sigma^{-1} \mathbf{c} \cap \mathbf{d}\right)}{\mu_{\nu}(\mathbf{d})}\right\},
\end{aligned}
$$

for $\mathrm{c}=\left[c_{0} \cdots c_{m-1}\right]$.

(3) \|\|$_{1}$ is a norm on column vectors with $\|V\|_{1}=\sum_{i}\left|V_{i}\right|$ if $V=\left(V_{i}\right)$, and also on matrices with $\|A\|_{1}=\sup _{j} \sum_{i}\left|a_{i j}\right|$ if $A=\left(a_{i j}\right)$.

Note that $\left\|V_{m}(\theta, \phi)\right\|_{1},\left\|A_{m}(\theta, \phi)\right\|_{1}=1$.

(4) For any $\phi$, and $\boldsymbol{\theta} \neq 0$ (regarded as real variables now), and $\frac{1}{2}<\delta \leq 1$, define, for a column vector-valued function $V(\theta, \phi)$,

$$
\begin{aligned}
\|V\|_{1, \boldsymbol{\theta}, \phi, \delta} & =\frac{\|V(\boldsymbol{\theta}, \boldsymbol{\phi})-V(\boldsymbol{0}, \boldsymbol{\phi})\|_{1}}{\left|\theta_{1}\right|^{2 \delta-1}+\cdots+\left|\theta_{v_{1}}\right|^{2 \delta-1}} \quad \text { if } \delta<1, \\
& =\frac{\|V(\boldsymbol{\theta}, \boldsymbol{\phi})-V(\mathbf{0}, \boldsymbol{\phi})\|_{1}}{\left|\theta_{1} \log \theta_{1}\right|+\cdots+\left|\theta_{v_{1}} \log \theta_{v_{1}}\right|} \quad \text { if } \delta=1,
\end{aligned}
$$

and similarly for a matrix-valued function $A(\theta, \phi)$.

The first stage in proving (2) of theorem 3, proposition 4, is proved as 3.3 of [6]:

Proposition 4. For any fixed $t, u$, if $m^{8 t+3} \leq k \leq m^{u}$,

$$
S_{k}+S_{k+1}=\frac{2}{(2 \pi)^{v}} \int_{\left[-1 / m^{\prime}, 1 / m^{\prime}\right]^{v}} W_{m}\left(A_{m}(\theta, \phi)^{k-m} V_{m}(\theta, \phi) d \theta d \phi+O\left(\eta^{m}\right),\right.
$$

some $\eta<1$. 
For the next stage, the following analogue of 4.2 of [6] is needed:

Proposition 5. Let $F: \mathbb{R}^{v_{1}} \times \mathbb{R}^{v_{2}} \times \mathbb{C} \times \mathbb{C}^{\varepsilon_{m}} \rightarrow \mathbb{C}^{\varepsilon_{m}} \times \mathbb{C}$ be defined by

$$
F(\boldsymbol{\theta}, \boldsymbol{\phi}, \lambda, \mathbf{y})=\left(\begin{array}{c}
\left(\lambda-A_{m}(\theta+\phi)\right)(\boldsymbol{\mu}+\mathbf{y}) \\
\sum_{\mathbf{c} \in e_{m}} y(\mathbf{c})
\end{array}\right),
$$

where $\mathbf{y}=(y(\mathbf{c}))$ and $\boldsymbol{\mu}=\left(\mu_{\nu}(\mathbf{c})\right)$, so $\boldsymbol{\mu}=V_{m}(\mathbf{0})$. Then there exist $C^{0}$ functions $\lambda_{m}(\boldsymbol{\theta}, \boldsymbol{\phi}), \mathbf{y}(\boldsymbol{\theta}, \boldsymbol{\phi})$ defined for $\left|\theta_{i}\right| \leq C / m^{2 /(2 \delta-1)}$ or (if $\left.\delta=1\right) \quad C /\left(m^{2} \log m\right)$, and $\left|\phi_{i}\right| \leq C / m^{2}$, with $F\left(\boldsymbol{\theta}, \boldsymbol{\phi}, \lambda_{m}(\boldsymbol{\theta}, \boldsymbol{\phi}), \mathbf{y}(\boldsymbol{\theta}, \boldsymbol{\phi})\right)=0$. Moreover, $\lambda_{m}, \mathbf{y}$ are $C^{\infty}$ in $\phi$, and

$$
\begin{aligned}
\left\|D_{\phi}^{k}\left(\begin{array}{c}
\lambda_{m} \\
\mathbf{y}
\end{array}\right)\right\|_{1} & \leq d_{k} m^{n_{k}} \\
\left\|D_{\phi}^{k}\left(\begin{array}{c}
\lambda_{m} \\
\mathbf{y}
\end{array}\right)\right\|_{1, \boldsymbol{\theta}, \boldsymbol{\phi}, \delta} & \leq d_{k} m^{n_{k}}
\end{aligned}
$$

for constants $d_{k}, n_{k}$. Moreover, if the sequence $\left(\begin{array}{c}\lambda_{m}^{r} \\ y^{r}\end{array}\right)$ is defined inductively by

$$
\begin{gathered}
\left(\begin{array}{c}
\lambda_{m}^{0}(\boldsymbol{\theta}, \boldsymbol{\phi}) \\
\mathbf{y}^{0}(\boldsymbol{\theta}, \boldsymbol{\phi})
\end{array}\right)=\left(\begin{array}{l}
1 \\
\mathbf{\theta}
\end{array}\right), \\
\left(\begin{array}{c}
\lambda_{m}^{r+1}(\boldsymbol{\theta}, \boldsymbol{\phi}) \\
\mathbf{y}^{r+1}(\boldsymbol{\theta}, \boldsymbol{\phi})
\end{array}\right)=\left(\begin{array}{c}
\lambda_{m}^{r}(\boldsymbol{\theta}, \boldsymbol{\phi}) \\
\mathbf{y}^{r}(\boldsymbol{\theta}, \boldsymbol{\phi})
\end{array}\right)-\left(D F_{\lambda_{m}^{r}, \mathbf{y}^{r}}\right)^{-1} F\left(\boldsymbol{\theta}, \boldsymbol{\phi}, \lambda_{m}^{r}, \mathbf{y}^{r}\right),
\end{gathered}
$$

then

$$
\left\|\left(\begin{array}{c}
\lambda_{m} \\
\mathbf{y}
\end{array}\right)-\left(\begin{array}{c}
\lambda_{m}^{1} \\
\mathbf{y}^{1}
\end{array}\right)\right\|_{1, \theta, 0, \delta} \leq d m^{4}\left(|\theta|^{2 \delta-1}+\cdots+\left|\theta_{V_{1}}\right|^{2 \delta-1}\right)
$$

for a constant $d$, where $\left|\boldsymbol{\theta}_{i}\right|^{2 \delta-1}$ is replaced by $\left|\theta_{i} \log \theta_{i}\right|$ if $\delta=1$.

Similar results hold for extending the eigenvalue 1 , and eigenvector $W_{m}$, of $\left(A_{m}(0)\right)^{T}$, with \|\|$_{\infty}$ norms, by exactly dual methods.

In order to prove proposition 5 , we need the following lemma and its corollary:

LEMMA 6.

$$
\begin{aligned}
\sum_{n=1}^{\infty} \frac{\mid \exp (\text { in } \theta)-1 \mid}{n^{2 \delta}} & \leq A|\theta|^{2 \delta-1} \quad \text { if } \frac{1}{2}<\delta<1 \\
& \leq \boldsymbol{A}|\theta \log \theta| \quad \text { if } \delta=1 .
\end{aligned}
$$

Proof. For any $\varepsilon>0$, if $[|\varepsilon / \theta|]$ denotes the integral part of $|\varepsilon / \theta|$,

Corollary 7.

$$
\begin{aligned}
\sum_{n=1}^{\infty} \frac{|\exp (i n \theta)-1|}{n^{2 \delta}} & \leq 4|\theta| \sum_{n=1}^{[l e / \theta \mid]} n^{1-2 \delta}+2 \sum_{[|\varepsilon / \theta|]+1}^{\infty} n^{-2 \delta} \\
& \leq A_{1}|\theta| \cdot|\theta|^{2 \delta-2}+A_{2}|\theta|^{2 \delta-1} \quad \text { if } \delta<1 \\
& \leq A_{1}|\theta||\log \theta|+A_{2}|\theta| \text { if } \delta=1 .
\end{aligned}
$$

$$
\begin{aligned}
\left\|V_{m}\right\|_{1, \theta, \phi, \delta} & \leq B m \\
\left\|D_{\phi}^{k} A_{m}\right\|_{1, \boldsymbol{\theta}, \boldsymbol{\phi}, \delta} & \leq A_{k} \quad(k \geq 0)
\end{aligned}
$$

for some constants $B, A_{k}$. 
Proof. This follows from lemmas 2, 6, and also the following property of a Gibbs measure (see [6], 1.7.2) for the bound on $\left\|V_{m}\right\|_{1, \boldsymbol{\theta}, \boldsymbol{\phi}, \delta}$ :

$$
\mu_{\nu}\left(\left[e_{0} \cdots e_{p-1}\right]\right) \leq D \mu_{\nu}\left(\left[e_{0} \cdots e_{r-1}\right]\right) \mu_{\nu}\left(\left[e_{r} \cdots e_{p-1}\right]\right),
$$

some constant $D$, any $r$.

Proof of proposition 5. As in (4.2) of [6],

$$
D F_{\lambda y}=\left(\begin{array}{cc}
\mu+y & \lambda-A_{m} \\
0 & 1 \cdots 1
\end{array}\right)
$$

As in (3.2) of [6],

$$
\left\|\left(I-A_{m}(\mathbf{0}, \mathbf{0})\right) V\right\|_{1} \geq\left(D^{1} / m\right)\|V\|_{1},
$$

some $D^{1}$, if $\sum_{\mathbf{c} \in \varepsilon_{m}} V(\mathbf{c})=0$, and hence (using corollary 7)

$$
\begin{gathered}
\left\|\left(D F_{1,0}\right)^{-1}\right\|_{1} \leq D m \quad \text { if }\left|\theta_{i}\right| \leq c / m^{1 /(2 \delta-1)}, \quad\left|\phi_{i}\right| \leq c / m \text { for } \delta<1, \\
\left|\theta_{i}\right| \leq c /(m \log m), \quad\left|\phi_{i}\right| \leq c / m \quad \text { if } \delta=1,
\end{gathered}
$$

for some constants $D, c$. Hence, for $(\boldsymbol{\theta}, \phi)$ in this set $\mathcal{U}$ say, $D F_{\lambda_{m^{\prime}}, y^{\prime}}$ is invertible if $\left|\lambda_{m}^{r}-1\right|,\left\|\mathbf{y}^{r}\right\|_{1} \leq 1 /(2 D m)$, and then $\left\|\left(D F_{\lambda_{m}^{r}, \mathbf{y}^{r}}\right)^{-1}\right\|_{1} \leq 2 D m$, so that $\lambda_{m}^{r+1}, \mathbf{y}^{r+1}$ are defined.

Fix a set $U_{1} \subseteq \mathcal{U}$, and let

$$
\begin{aligned}
\varepsilon_{0} & =\sup _{(\boldsymbol{\theta}, \phi) \in \mathscr{U}_{1}}\left\|F\left(\boldsymbol{\theta}, \phi, \lambda_{m}^{0}, \mathbf{y}^{0}\right)\right\|_{1} \quad\left(\operatorname{recall}\left(\lambda_{m}^{0}, \mathbf{y}^{0}\right)=(1,0)\right) \\
& =\sup _{(\boldsymbol{\theta}, \phi) \in \mathscr{q}_{U_{1}}}\left\|\left(I-A_{m}(\boldsymbol{\theta}, \phi)\right) \mu\right\|_{1} .
\end{aligned}
$$

So from corollary 7 , by suitable choice of $\mathcal{U}_{1}, \varepsilon_{0}$ can be made arbitrarily small. As in (4.2) of [6], we have inductively that:

$$
\begin{array}{r}
\sup _{(\theta, \phi) \in \mathscr{U}_{1}}\left\|F\left(\boldsymbol{\theta}, \boldsymbol{\phi}, \lambda_{m}^{r}, \mathbf{y}^{r}\right)\right\|_{1} \leq(2 D m)^{2 r-1} \varepsilon_{0}^{2 r} \\
\sup _{(\theta, \phi) \in \mathcal{U}_{1}}\left\|\begin{array}{c}
\lambda_{m}^{r}-\lambda_{m}^{r-1} \\
y^{r}-y^{r-1}
\end{array}\right\|_{1} \leq\left(2 D m \varepsilon_{0}\right)^{2 r-1} .
\end{array}
$$

So by suitable choice of $\mathcal{U}_{1}$ (in fact, the choice indicated in the statement of the proposition) we may assume that $\lambda_{m}^{r}, \mathbf{y}^{r}$ exist for all $r,\left|\lambda_{m}^{r}-1\right|\left\|\mathbf{y}^{r}\right\|_{1} \leq 1 /(2 D m)$, and the sequence $\left(\begin{array}{c}\lambda_{m}^{r} \\ \mathbf{y}^{r}\end{array}\right)$ converges uniformly to $\left(\begin{array}{c}\lambda_{m} \\ \mathbf{y}\end{array}\right)$ which solves $F\left(\boldsymbol{\theta}, \boldsymbol{\phi}, \lambda_{m}(\boldsymbol{\theta}, \boldsymbol{\phi}), \mathbf{y}(\boldsymbol{\theta}, \boldsymbol{\phi})\right)=0$.

The bound on $\left\|D_{\phi}^{k}\left(\begin{array}{c}\lambda_{m} \\ \mathbf{y}\end{array}\right)\right\|_{1}$ then follows from the repeated differentiation of

$$
D_{\phi}\left(\begin{array}{c}
\lambda_{m} \\
\mathbf{y}
\end{array}\right)=\left(D F_{\lambda_{m}, y}\right)^{-1}\left(\left(D_{\phi} A_{m}(\theta, \phi)\right)(\mu+\mathbf{y})\right)
$$

using corollary 7 and the bound $\left\|\left(D F_{\lambda_{m}, y}\right)^{-1}\right\|_{1} \leq 2 D m$.

It remains to compute \|\|$_{1, \theta, \phi, \delta}$-seminorms. First,

$$
\left\|D F_{\lambda_{m}^{r}, \mathbf{y}^{r}}\right\|_{1, \boldsymbol{\theta}, \boldsymbol{\phi}, \delta} \leq\left\|\left(\begin{array}{c}
\lambda_{m}^{r} \\
\mathbf{y}^{r}
\end{array}\right)\right\|_{1, \boldsymbol{\theta}, \boldsymbol{\phi}, \delta}+\left\|A_{m}\right\|_{1, \boldsymbol{\theta}, \boldsymbol{\phi}, \delta}
$$


Hence, from the definition of $\left(\begin{array}{l}\lambda_{m}^{r+1} \\ y^{r+1}\end{array}\right)$,

$$
\begin{gathered}
\left\|\left(\begin{array}{c}
\lambda_{m}^{r+1}-\lambda_{m}^{r} \\
\mathbf{y}^{r+1}-\mathbf{y}^{r}
\end{array}\right)\right\|_{1, \boldsymbol{\theta}, \boldsymbol{\phi}, \delta} \\
\leq 2 D m\left\|F\left(\lambda_{m}^{r}, \mathbf{y}^{r}\right)\right\|_{1, \boldsymbol{\theta}, \boldsymbol{\phi}, \delta}+\left\|\left(D F_{\lambda_{m}, \mathbf{y}^{r}}\right)^{-1}\right\|_{1, \boldsymbol{\theta}, \boldsymbol{\phi}, \delta}\left\|F\left(\lambda_{m}^{r}, \mathbf{y}^{r}\right)\right\|_{1} \\
\leq 2 D m\left\|F\left(\lambda_{m}^{r}, \mathbf{y}^{r}\right)\right\|_{1, \boldsymbol{\theta}, \boldsymbol{\phi}, \delta} \\
+(2 D m)^{2}\left(\left\|\left(\begin{array}{c}
\lambda_{m}^{r} \\
\mathbf{y}^{r}
\end{array}\right)\right\|_{1, \boldsymbol{\theta}, \boldsymbol{\phi}, \delta}+\left\|A_{m}\right\|_{1, \boldsymbol{\theta}, \boldsymbol{\phi}, \delta}\right)\left\|F\left(\lambda_{m}^{r}, \mathbf{y}^{r}\right)\right\|_{1} .
\end{gathered}
$$

Since $F$ is quadratic in $\lambda, \mathbf{y}$, the Taylor expansion of

$$
F\left(\left(\begin{array}{c}
\lambda_{m}^{r-1} \\
\mathbf{y}^{r-1}
\end{array}\right)+\left(\begin{array}{c}
\lambda_{m}^{r}-\lambda_{m}^{r-1} \\
\mathbf{y}^{r}-\mathbf{y}^{r-1}
\end{array}\right)\right)
$$

is particularly simple, and we obtain

$$
\begin{aligned}
& \left\|F\left(\lambda_{m}^{r}, \mathbf{y}^{r}\right)\right\|_{1, \boldsymbol{\theta}, \boldsymbol{\phi}, \boldsymbol{\delta}} \\
& \leq C_{1}\left\|\left(D F_{\lambda_{m}^{r-1}, \mathbf{y}^{r-1}}\right)^{-1} F\left(\lambda_{m}^{r-1}, \mathbf{y}^{r-1}\right)\right\|_{1,0, \Phi, \delta}\left\|\left(D F_{\lambda_{m}^{r-1}, y^{r-1}}\right)^{-1} F\left(\lambda_{m}^{r-1}, \mathbf{y}^{r-1}\right)\right\|_{1} \\
& \leq C_{2} m\left\|\left(\begin{array}{c}
\lambda_{m}^{r}-\lambda_{m}^{r-1} \\
\mathbf{y}^{r}-\mathbf{y}^{r-1}
\end{array}\right)\right\|_{1,0, \phi, \delta}\left\|F\left(\lambda_{m}^{r-1}, \mathbf{y}^{r-1}\right)\right\|_{1} .
\end{aligned}
$$

Substituting from (1) and (4), (3) becomes

$$
\begin{gathered}
\left\|\begin{array}{c}
\lambda_{m}^{r+1}-\lambda_{m}^{r} \\
\mathbf{y}^{r+1}-\mathbf{y}^{r}
\end{array}\right\|_{1, \boldsymbol{\theta , \boldsymbol { \phi } , \delta}} \\
\leq C_{3} m\left(2 D m \varepsilon_{0}\right)^{2 r-1}\left(\left\|\boldsymbol{A}_{m}\right\|_{1, \boldsymbol{\theta , \phi}, \delta}+\left\|\left(\begin{array}{c}
\lambda_{m}^{r} \\
\mathbf{y}^{r}
\end{array}\right)\right\|_{1, \mathbf{\theta , \boldsymbol { \phi } , \delta}}+\left\|\left(\begin{array}{c}
\lambda_{m}^{r}-\lambda_{m}^{r-1} \\
\mathbf{y}^{r}-\mathbf{y}^{r-1}
\end{array}\right)\right\|_{1, \boldsymbol{\theta}, \boldsymbol{\phi}, \delta}\right) .
\end{gathered}
$$

Inductively we can prove:

$$
\left\|\left(\begin{array}{c}
\lambda_{m}^{r+1}-\lambda_{m}^{r} \\
\mathbf{y}^{r-1}-\mathbf{y}^{r}
\end{array}\right)\right\|_{1, \mathbf{\theta , \phi , \delta}} \leq C_{4} m^{3}\left(2 D m \varepsilon_{0}\right)^{2 r-1} \quad \text { if } r \geq 1
$$

provided that

$$
\left\|A_{m}\right\|_{1, \theta, \phi, \delta}+\left\|\left(\begin{array}{c}
\lambda_{m}^{1} \\
y^{1}
\end{array}\right)\right\|_{1, \theta, \phi, \delta}+2 \sum_{r=0}^{\infty} C_{4} m^{3}\left(2 D m \varepsilon_{0}\right)^{2 r} \leq\left(C_{4} / C_{3}\right) m^{2}
$$

This can be arranged for $C_{4}$ large enough, and $\varepsilon_{0}=C / \mathrm{m}^{2}, C$ small enough, since

(a) $\left\|A_{m}\right\|_{1, \boldsymbol{\theta}, \phi, \delta}$ is bounded, by corollary 7 ;

(b) $\left\|\left(\begin{array}{c}\lambda_{m}^{1} \\ \mathbf{y}^{1}\end{array}\right)\right\|_{1, \boldsymbol{\theta}, \boldsymbol{\phi}, \delta}=\left\|\left(\begin{array}{c}\lambda_{m}^{1}-\lambda_{m}^{0} \\ \mathbf{y}^{1}-\mathbf{y}^{0}\end{array}\right)\right\|_{1, \boldsymbol{\theta}, \boldsymbol{\phi}, \delta}=\left\|\left(D F_{1,0}\right)^{-1} F(\cdots 1,0)\right\|_{1, \boldsymbol{\theta}, \boldsymbol{\phi}, \delta}$

$$
\begin{gathered}
\leq 2 D m\|F(\cdots 1, \mathbf{0})\|_{1, \boldsymbol{\theta}, \boldsymbol{\phi}, \delta}+(2 D m)^{2}\left\|D F_{1, \boldsymbol{0}}\right\|_{1, \boldsymbol{\theta}, \boldsymbol{\phi}, \delta}\left\|\left(I-A_{m}(\boldsymbol{\theta}, \boldsymbol{\phi})\right) \boldsymbol{\mu}\right\|_{1} \\
\leq C_{5} m^{2}, \text { by corollary } 7 .
\end{gathered}
$$


The bound on $\left\|\lambda_{m}-\lambda_{m}^{1}\right\|_{1, \theta, 0, \delta}$ follows from (6) since, by corollary 7 , and the definition of $\varepsilon_{0}$, if $U_{1}=\left\{\left(\theta^{1}, 0\right):\left|\theta_{i}^{1}\right| \leq\left|\theta_{i}\right|\right\}$ then

$$
\begin{gathered}
\varepsilon_{0} \leq C_{6}\left(\left|\theta_{1}\right|^{2 \delta-1}+\cdots+\left|\theta_{v_{1}}\right|^{2 \delta-1}\right) \quad \text { if } \delta<1 \\
\varepsilon_{0} \leq C_{6}\left(\left|\theta_{1} \log \theta_{1}\right|+\cdots+\left|\theta_{v_{1}} \log \theta_{v_{1}}\right|\right) \quad \text { if } \delta=1 .
\end{gathered}
$$

As for $\left\|D_{\phi}^{k}\left(\begin{array}{c}\lambda_{m} \\ y\end{array}\right)\right\|_{1}$, the bounds on $\left\|D_{\phi}^{k}\left(\begin{array}{c}\lambda_{m} \\ y\end{array}\right)\right\|_{1, \theta, \phi, \delta}$ follow from differentiating (2).

Proposition 5 is used to prove the following corollaries:

COROLlar y 8 (analogue of (4.4) of [6]). For any sufficiently large $t$, independent of $m$, if $m^{8 t+3} \leq k \leq m^{n}$,

$$
\begin{aligned}
S_{k}+S_{k+1}=2 & (1+O(1 / m)) \frac{1}{(2 \pi)^{v}} \int_{\left[-1 / m^{t}, 1 / m^{t}\right]^{v}}\left(\lambda_{m}(\theta, \phi)\right)^{k-m} d \theta d \phi \\
& +O\left(\eta^{m}\right), \text { some } \eta<1 .
\end{aligned}
$$

Proof. Exactly as in (4.4) of [6], using the decomposition, for $\boldsymbol{\theta}$, $\boldsymbol{\phi}$ near $\boldsymbol{0}$, $\mathbb{R}^{\varepsilon_{m}}=\operatorname{Im} \boldsymbol{P}_{m}(\boldsymbol{\theta}, \boldsymbol{\phi}) \oplus \operatorname{Ker} \boldsymbol{P}_{m}(\boldsymbol{\theta}, \boldsymbol{\phi})$, where $\boldsymbol{A}_{m}(\boldsymbol{\theta}, \boldsymbol{\phi})$ has eigenvalue $\lambda_{m}(\boldsymbol{\theta}, \boldsymbol{\phi})$ on $\operatorname{Im} P_{m}(\theta, \phi)$, and $\left\|\left(A_{m}(\theta, \phi)\right)^{m+s}\right\|_{1}<\beta<1$ on the $A_{m}(\theta, \phi)$-invariant subspace $\operatorname{Ker} P_{m}(\theta, \phi)$, some $s, \beta$ independent of $m$ ((3.2) of [6] is used here). The Hölder continuity of $\lambda_{m}, P_{m}$ at 0 established in proposition 5 (the dual results of proposition 5 for $A_{m}^{T}$ are needed to prove Hölder continuity of $P_{m}$ ) are enough for the proof.

Note. As in (4.1) of [6], $\lambda_{m}(\boldsymbol{\theta}, \phi)$ is real, so the first $\phi$-derivatives of $\lambda_{m}$ vanish at $(\boldsymbol{\theta}, \boldsymbol{\phi})=(\mathbf{0}, \mathbf{0})$.

COROLLARY 9 (immediate from proposition 5).

$$
\begin{aligned}
\lambda_{m}(\boldsymbol{\theta}, \boldsymbol{\phi})= & \lambda_{m}^{1}(\boldsymbol{\theta}, \mathbf{0})+\frac{1}{2}\left(\phi_{1} \cdots \phi_{v_{2}}\right)\left(\frac{\partial^{2} \lambda_{m}(\mathbf{0})}{\partial \phi_{i} \partial \phi_{j}}\right)\left(\begin{array}{c}
\phi_{1} \\
\phi_{v_{2}}
\end{array}\right) \\
& +O\left(m^{4}\left(\left|\theta_{1}\right|^{2 \delta-1}+\cdots+\left|\theta_{v_{1}}\right|^{2 \delta-1}\right)^{2}\right)+O\left(\sum_{i, j} m^{n_{1}}\left|\theta_{i}\right|^{2 \delta-1}\left|\phi_{j}\right|\right) \\
& +O\left(\sum_{i, j} m^{n_{2}}\left|\theta_{i}\right|^{2 \delta-1}\left|\phi_{j}\right|^{2}\right)+O\left(\sum_{j} m^{n_{3}}\left|\phi_{j}\right|^{3}\right)
\end{aligned}
$$

with $\left|\theta_{i}\right|^{2 \delta-1}$ replaced by $\left|\theta_{i}\right|\left|\log \theta_{i}\right|$ if $\delta=1$.

Note. The aim is to show $\lambda_{m}^{1}(\boldsymbol{\theta}, \mathbf{0})=1-O\left(\left|\theta_{1}\right|^{2 \delta-1}+\cdots+\left|\theta_{v_{1}}\right|^{2 \delta-1}\right)$. For exactly as in (4.6) of [6], $\left(\phi_{1} \cdots \phi_{v_{2}}\right)\left(\frac{\partial^{2} \lambda_{m}(0)}{\partial \phi_{i} \partial \phi_{j}}\right)\left(\begin{array}{l}\phi_{1} \\ \phi_{v_{2}}\end{array}\right)$ is boundedly negative definite of rank $v_{2}$ (we can reduce to the case of a finite symbol space by putting $\theta=0$ and replacing $\left\{c_{i}^{n}\right\}_{n>0}$, $\left\{c_{i}^{-n}\right\}_{n<0}$ by single symbols). It is then not hard to see that $\lambda_{m}^{1}(\theta, 0)+$ $\frac{1}{2}\left(\phi_{1} \cdots \phi_{v_{2}}\right)\left(\frac{\partial^{2} \lambda_{m}(0)}{\partial \phi_{i} \partial \phi_{j}}\right)\left(\begin{array}{l}\phi_{1} \\ \phi_{v_{2}}\end{array}\right)$ is the dominating part of $\lambda_{m}(\theta, \phi)$ (in spite of the $\left|\theta_{i} \log \theta_{i}\right|$ terms when $\delta=1$ ).

Calculation of $\lambda_{m}^{1}(\boldsymbol{\theta}, \mathbf{0})$. By definition,

$$
\lambda_{m}^{1}(\boldsymbol{\theta}, \mathbf{0})=1-\left(D F_{1,0}(\boldsymbol{\theta}, \mathbf{0})\right)^{-1}\left(\begin{array}{c}
\left(I-A_{m}(\boldsymbol{\theta}, \mathbf{0})\right) \boldsymbol{\mu} \\
0
\end{array}\right) .
$$


It can be checked that $\left(D F_{1,0}\right)^{-1}$ is of the form $\left(\begin{array}{cc}1 \cdots 1 & 0 \\ B_{m}(\theta, \phi) & \mu\end{array}\right)$, where, if $M=$ $(\mu \cdots \mu)\left(a\right.$ matrix with rows and columns indexed by $\left.\varepsilon_{m}\right)$, then

$$
\begin{aligned}
B_{m}(\boldsymbol{\theta}, \boldsymbol{\phi})\left(I-A_{m}(\boldsymbol{\theta}, \boldsymbol{\phi})\right) & =\left(I-A_{m}(\boldsymbol{\theta}, \boldsymbol{\phi})\right) B_{m}(\boldsymbol{\theta}, \boldsymbol{\phi})=I-M \\
& =\text { projection on }\left\{(y(\mathbf{c})): \sum_{\mathbf{c} \in \varepsilon_{m}} y(\mathbf{c})=0\right\} \text { along sp }(\boldsymbol{\mu}) .
\end{aligned}
$$

$\left(B_{m}(\theta, \phi)\right.$ does exist, since by $\S 3$ of $[6]\left\|A_{m}(0,0)\right\|_{1}<1$ on $\operatorname{Im}(I-M)$, hence also for nearby $(\boldsymbol{\theta}, \boldsymbol{\phi})$.) So

$$
\begin{aligned}
\lambda_{m}^{1}(\boldsymbol{\theta}, \mathbf{0}) & =1-(1 \cdots 1)\left(I-A_{m}(\boldsymbol{\theta}, \boldsymbol{0})\right) \mu \\
& =2 \sum_{i=1}^{r} \sum_{n=1}^{\infty} \mu_{\nu}\left(\left[c_{i}^{n}\right]\right) \cos n \theta\left(c_{i}\right)
\end{aligned}
$$

(using the fact that $\left.\mu_{\nu}\left(\left[c_{i}^{n}\right]\right)=\mu_{\nu}\left(\left[c_{i}^{-n}\right]\right)\right)$.

Hence, from lemma 2,

$$
\lambda_{m}^{1}(\theta, 0)=2 \sum_{i=1}^{\infty} b_{i} \sum_{n=1}^{\infty} \frac{1}{n^{2 \delta}} \cos n \theta\left(c_{i}\right)+O\left(\left|\theta\left(c_{1}\right)\right|+\cdots+\left|\theta\left(c_{r}\right)\right|\right),
$$

since any cosine series with $n$th term $O\left(1 / n^{2 \delta+1}\right)\left(\delta>\frac{1}{2}\right)$ is $C^{1}$ with first derivative at 0 vanishing.

LEMMA 10 . If

$$
f(\theta)=\sum_{n=1}^{\infty} \frac{\cos n \theta}{n^{2 \delta}}, \quad f(\theta)=\sum_{n=1}^{\infty} \frac{1}{n^{2 \delta}}\left(1-C|\theta|^{2 \delta-1}+O\left(|\theta|^{2 \delta-1}\right)\right)
$$

for some constant $C>0\left(\delta>\frac{1}{2}\right)$.

Proof. This is standard complex analysis. Since, clearly, $f(\theta)=f(-\theta)$, we need only consider the expansion for $\theta>0$. If

$$
0 \leq \theta \leq 2 \pi, \quad f(\theta)=\lim _{N \rightarrow \infty} \frac{1}{2 i} \int_{\gamma_{N}} \frac{\cos (\pi-\theta) z}{z^{2 \delta} \sin \pi z} d z
$$

where $\gamma_{N}$ is the contour shown in figure 3. In the limit as $N \rightarrow \infty$, the integral vanishes except on the imaginary axis and half-circle. If $\delta=1$, the imaginary axis integral also cancels out, and the integral around the half-circle, which is a $C^{\infty}$ function, has derivative $-\frac{1}{2} \pi$ at 0 . If $\frac{1}{2}<\delta<1$,

$$
f(\theta)=g(\theta)-\sin \pi \delta \int_{0}^{\infty} \frac{1-\exp (-y \theta)}{y^{2 \delta}} d y, \quad \text { where } g \text { is } C^{\infty} .
$$

But

$$
\int_{0}^{\infty} \frac{1-\exp (-y \theta)}{y^{2 \delta}} d y=\theta^{2 \delta-1} \int_{0}^{\infty} \frac{1-\exp (-y)}{y^{2 \delta}} d y \quad \text { by change of variable. }
$$

Completion of the proof of theorem 3. It has now been proved that

$$
\lambda_{m}(\theta, \phi)=1-\sum_{i=1}^{\infty} a_{i}\left|\theta\left(c_{i}\right)\right|^{2 \delta-1}+\frac{1}{2}\left(\phi_{1} \cdots \phi_{v_{2}}\right)\left(\frac{\partial^{2} \lambda_{m}(\boldsymbol{0})}{\partial \phi_{i} \partial \phi_{j}}\right)\left(\begin{array}{c}
\phi_{1} \\
\phi_{v_{2}}
\end{array}\right)+\text { higher order terms, }
$$




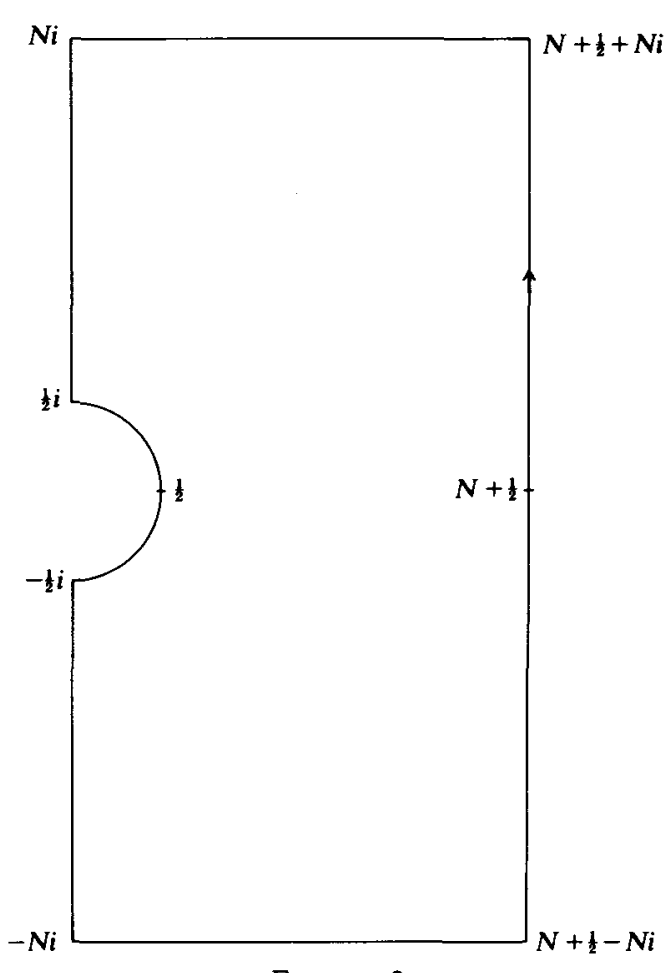

Figure 3

for constants $a_{i}>0$, where $-\frac{1}{2}\left(\phi_{1} \cdots \phi_{v_{2}}\right)\left(\frac{\partial^{2} \lambda_{m}(\mathbf{0})}{\partial \phi_{i} \partial \phi_{i}}\right)\left(\begin{array}{c}\phi_{1} \\ \phi_{v_{2}}\end{array}\right)$ converges geometrically fast to a function $G\left(\phi_{1} \cdots \phi_{v_{2}}\right)$, where $G$ is boundedly positive definite (from [6], (4.6)). So from corollary 8 ,

$$
\begin{aligned}
S_{k}+S_{k+1}= & \frac{2}{(2 \pi)^{v}}\left(1+O\left(\frac{1}{m}\right)\right) \int_{\left[-1 / m^{t}, 1 / m^{t}\right]^{v}}\left(\operatorname { e x p } \left\{-(k-m)\left(\sum_{i=1}^{r} a_{i}\left|\theta\left(c_{i}\right)\right|^{2 \delta-1}\right)\right.\right. \\
& \left.\left.+G\left(\phi_{1} \cdots \phi_{v_{2}}\right)+\text { higher order terms }\right\}\right) d \theta d \phi+O\left(\eta^{m}\right), \text { some } \eta<1 .
\end{aligned}
$$

Change of variable then gives

$$
\begin{aligned}
S_{k}+S_{k+1} & \sim \frac{2}{(2 \pi)^{v}} k^{-\left(v_{1} /(2 \delta-1)+\frac{1}{2} v_{2}\right)} \int_{\mathbb{R}^{v}} \exp \left\{-\left(\sum_{i=1}^{r} a_{i}\left|\theta\left(c_{i}\right)\right|^{2 \delta-1}\right.\right. \\
& \left.\left.+G\left(\phi_{1} \cdots \phi_{v_{2}}\right)\right)\right\} d \boldsymbol{\theta} d \boldsymbol{\phi}
\end{aligned}
$$

and theorem 3 is proved.

The author was supported by a junior research fellowship at St Hilda's College, Oxford, England.

\section{REFERENCES}

[1] A. F. Beardon. The exponent of convergence of Poincaré series, Proc. London Math. Soc. (3) 18 (1968), 461-483. 
[2] T. Lyons \& H. P. McKean. The winding of plane Brownian motion. Advances in Math. (in the press).

[3] H. P. McKean \& D. Sullivan. In preparation.

[4] M. Morse. Symbolic Dynamics (notes by R. Oldenburger). I.A.S.: Princeton, 1966.

[5] S. J. Patterson. The limit set of a Fuchsian group. Acta Math. 136 (1976), 241-273.

[6] M. Rees. Checking ergodicity of some geodesic flows with infinite Gibbs measure. Ergod. Th. and Dynam. Sys. 1 (1981), 107-133.

[7] C. Series. Symbolic dynamics for geodesic flows. Acta Math. (in the press).

[8] D. Sullivan. The density at infinity of a discrete group of hyperbolic motions. IHES Publ. Math. 50 (1979), 171-202. 